\title{
Neonatal exposure to active pulmonary tuberculosis in a health care professional
}

\section{Mithu Sen, Daniel Gregson, James Lewis}

\section{Abstract}

NOSOCOMIAL TRANSMISSION OF TUBERCULOSIS (TB) is a recognized risk. Although many outbreaks of TB in health care settings have been reported, there are few cases of nosocomial transmission to neonates. We report our experience in investigating and managing the exposure over 16 days of 124 neonates, 301 visitors and 219 health care workers to a health care worker with active TB in a neonatal intensive care unit.

CMAJ 2005;172(11):1453-6

$\mathrm{N}$ osocomial transmission of tuberculosis (TB) has increased significantly over the last decade. ${ }^{1}$ However, there are few reported cases of such transmission to neonates. ${ }^{1}$ This is fortunate, since neonatal TB is associated with a high mortality. ${ }^{2,3}$ After the practice of removing infants from mothers with active pulmonary TB was introduced, neonatal mortality declined significantly. ${ }^{4}$

Currently, in developed countries, despite the availability of effective pharmacologic therapy, there are few established guidelines for the surveillance and management of neonates after exposure to an active case of TB. We report the results of our investigation of the neonates, visitors and health care workers who were exposed to a health care professional with active TB who worked in our neonatal intensive care unit (NICU).

\section{The index case}

A 39-year-old Burmese NICU physician with a known positive tuberculin skin test emigrated to Canada on July 1, 1997. Chest radiography in July 1997, conducted as part of his pre-immigration screening, was reported to be normal. He had never received therapy for latent TB infection. He began work at the NICU on July 1, 1998 (Box 1).

On July 20, 1998, the physician reported a fever and a productive cough, for which he sought medical attention. He began taking broad spectrum antibiotics, but his condition did not improve, and he stopped working in the NICU on July 25 . Chest radiography on July 27 showed right upper lung pneumonia, and sputum samples were taken on Aug. 1. A sputum smear was found to be positive for acid-fast bacilli the next day, and on Aug. 19 the diagnosis was confirmed when the sputum culture was positive for Mycobacterium tuberculosis.

To ensure that all possible contacts were included when determining the possible duration of exposure, and taking the physician's work schedule into account, we included the 11 days before the index case exhibited symptoms as well as the 5 days that he was ill but continued to work.

\section{Epidemiologic investigations and public health actions}

We categorized visitors and neonates who might have been exposed to the index case as being at high risk (exposed without respiratory precautions to the index case during the 5-day period when he was symptomatic) or at low risk (exposed during the 11-day period when he was asymptomatic) of infection. Infants were also considered to be at low risk if they were not directly exposed to the index case or if they were receiving mechanical ventilation throughout the 16-day exposure period. All of the health care workers were considered to be at high risk.

Box 1: Timeline for index case

- July 1, 1997: Arrives in Canada; pre-immigration results of chest radiography screening for tuberculosis reported to be normal

- July 1, 1998: Starts work in the neonatal intensive care unit

- July 20, 1998: Reports a fever and productive cough, for which he is prescribed antibiotics

- July 25, 1998: Stops working

- July 27, 1998: Chest radiography shows right upper lung pneumonia

- Aug. 1, 1998: Sputum samples taken

- Aug. 2, 1998: Sputum smear is positive for acid-fast bacilli

- Aug. 19, 1998: Sputum culture is positive for Mycobacterium tuberculosis 
After reviewing staffing records (including housekeeping staff, residents and students) and patient and visitor lists, we identified 219 health care workers, 124 neonates and 301 visitors, including parents, who might have been exposed to the index case.

We attempted to contact all of these people to advise them of the risk and to encourage them to undergo a tuberculin skin test. People with a previously positive tuberculin skin test result were excluded from further investigation. People with a newly positive skin test result were referred for further assessment, invited to have chest radiography and then assessed for or given therapy for latent TB infection. People whose skin test result was negative were asked to undergo a follow-up test in 3 months. ${ }^{5}$

\section{Health care workers}

Of the 219 health care workers who were identified as having potential exposure to the index case over the entire 16-day exposure period, 15 had previously had a positive tuberculin skin test result and were referred to a respirologist for further assessment (Table 1). All of the 15 had chest radiography, and none showed active disease. Because there was no documentation of the positive skin test result in the staff health records of 2 of the 15, they were assumed to be newly positive. Both began taking, as prophylaxis, isonicotinic acid hydrazide, commonly known as isoniazid. Only these 2 people were deemed to require isoniazid therapy immediately. At 3 months, 179 of the 204 people who had a known negative skin test result underwent testing. The remaining 25 refused the follow-up skin test, some because

\section{Table 1: Results of tuberculin skin tests at baseline and at 3- month follow-up for groups that were exposed or potentially exposed to the index case}

\begin{tabular}{|c|c|c|c|c|}
\hline \multirow[b]{3}{*}{ Group } & \multicolumn{3}{|c|}{ Results } & \multirow[b]{3}{*}{ Not tested } \\
\hline & \multicolumn{2}{|c|}{ Positive } & \multirow{2}{*}{$\frac{\text { Negative }}{\text { Known }}$} & \\
\hline & Known & New & & \\
\hline \multicolumn{5}{|c|}{ Health care workers $(n=219)^{*}$} \\
\hline Baseline & 13 & 2 & 204 & 0 \\
\hline Follow-up & 15 & 0 & 179 & 25 \\
\hline \multicolumn{5}{|c|}{ High-risk visitors $(n=133)$} \\
\hline Baseline & 0 & 2 & 109 & 22 \\
\hline Follow-up & 2 & 0 & 114 & 17 \\
\hline \multicolumn{5}{|c|}{ Low-risk visitors $(n=168)$} \\
\hline Baseline & 0 & 1 & 15 & 152 \\
\hline Follow-up & 1 & 4 & 148 & 15 \\
\hline \multicolumn{5}{|c|}{ High-risk neonates $(n=50)$} \\
\hline Baseline & 0 & 0 & 3 & 47 \\
\hline Follow-up & 0 & 0 & 45 & 5 \\
\hline \multicolumn{5}{|c|}{ Low-risk neonates $(n=74)$} \\
\hline Baseline & 0 & 0 & 72 & 2 \\
\hline Follow-up & 0 & 0 & 72 & 2 \\
\hline
\end{tabular}

*All of the staff were considered to be at high risk of infection. they were afraid of the potential adverse effects of isoniazid. All of the 25 were referred to a respirologist and received counselling about the risk of illness if they had been infected. Those who refused follow-up testing were not excluded from work.

\section{Visitors to the NICU}

Visitors who were considered to be at high risk of infection were more likely than visitors who were considered to be at low risk to accept tuberculin skin testing (111/133 [83.5\%] v. 16/168 [9.5\%] respectively).

Of the 133 high-risk visitors at baseline, 22 were not tested, either because they could not be traced or because they refused testing, 109 had a negative skin test result and 2 had a newly positive skin test result and normal chest radiography. One of the 2 with a positive skin test result, an immigrant from a country with a high incidence of $\mathrm{TB}$, received isoniazid therapy, and the other refused therapy. At 3 months, 114 high-risk visitors had follow-up skin tests, none of which was positive. The status of the remaining 17 people remained unknown, either because they were lost to follow-up or because they refused testing.

Only 1 of the 16 low-risk visitors who underwent a tuberculin skin test at baseline had a positive test result; 152 low-risk visitors were not tested. This person had immigrated from an area in which TB is endemic and had visited that area within the past year. Because there was no evidence of active disease, the person received therapy for latent TB infection. At 3 months, however, all 152 of the remaining low-risk visitors were contacted and tested, and 4 had a positive test result. Of these, 3 received therapy for latent TB infection. Contraindications to isoniazid were present in the fourth person.

Of the 7 visitors, both high and low risk, whose skin test result was ultimately positive for infection, active pulmonary TB was ruled out in all cases. Five received therapy for latent TB infection, 1 refused therapy and 1 was unable to take isoniazid because of contraindications.

\section{Neonatal patients}

Of the 50 neonates in the NICU who were exposed to the index case during the 5 days that he exhibited symptoms, baseline skin tests were performed on only 3 , the results of which were negative (Table 1). The remaining 47 infants were either too young (less than 3 months old) to obtain an accurate result $(n=43)$ or were discharged from the NICU before TB was diagnosed in the index case $(n=4)$. A total of 45 high-risk infants were tested at 3 months of age, and all of them had negative results. Of the remaining 5 infants, 3 were lost to follow-up and 2 were followed clinically by their physicians for signs and symptoms of infection after their caregivers refused to allow testing.

Because all exposed neonates are considered to be at 
risk of acquiring active $\mathrm{TB}$, we urged parents to accept prophylaxis at baseline, including the parents of the 3 neonates whose baseline skin test result was negative. The parents of 36 accepted, and the infants received therapy for latent TB infection (isoniazid [10 $\mathrm{mg} / \mathrm{kg}$ per day] and vitamin $\mathrm{B}_{6}$ supplements). ${ }^{6,7}$ Therapy was discontinued at 3 months of age because the repeat skin test results were negative in all cases. Although not specifically evaluated, no infant showed clinical evidence of hepatotoxicity. The remaining 14 neonates did not receive prophylaxis for various reasons, including mechanical ventilation during exposure $(n=3)$, refusal by parent or physician $(n=5)$, contraindications to isoniazid because of elevated liver enzyme levels $(n=2)$, minimal exposure due to specific circumstances $(n=2)$ and discharge from the NICU $(n=2)$ at the time of assessment.

Of the 74 low-risk neonates, all but 2 were tested and had negative results at baseline and at 3-month followup. The caregivers of the remaining 2 refused testing. No infant in the low-risk neonatal group was given isoniazid therapy.

\section{Comments}

Our investigation of 124 neonates, 301 visitors and 219 health care workers did not identify any newly positive skin test results caused by exposure to the index case. (The index case's spouse was also tested, with a positive result. Because no previous skin test result was known and chest radiography was normal, she was given isoniazid therapy.) Although there is a risk of hepatotoxicity when neonates are given isoniazid, the infants in our study, in accordance with those of earlier studies, did not show hepatotoxicity.

Light and associates reported the exposure of 437 neonates to a nurse's aide with active pulmonary TB. ${ }^{8}$ As was seen in our study, none of the infants had a positive skin test result at 3-month follow-up, and isoniazid therapy was discontinued. Of the 130 health care workers in that study who were exposed to the index case and tested, 4 (3.1\%) had an initial positive result, and none of the remaining 126 had a positive test result at 3 months. The transmission rate was similar to that of our study. However, in a study by Steiner and associates, 2 of 1668 neonates exposed to a case with active TB were infected. ${ }^{9}$

The rationale for treating latent TB infection in all exposed neonates stems from their immature cellular and humoral immune systems until 6-8 weeks of age. ${ }^{10,11}$ The neonatal lung is either deficient in macrophages or has macrophages with impaired phagocytosis. ${ }^{10,11}$ Moreover, impaired cell-mediated immunity may produce falsenegative skin test results.

At our institution, hospital employees are tested annually for TB, especially if they work in high-risk areas such as intensive care units. Some people working in the NICU were not screened because they did not comply with the regulations or because they refused testing. Because the index case had a positive tuberculin skin test result, he was further screened with chest radiography, the findings of which were normal. In retrospect, he should have received therapy for latent TB infection when his first skin test result was positive, but this did not happen.

There are several limitations to our study. Several people were not tested for infection at baseline, and therefore their skin test status was not known. Moreover, there were delays in contacting and testing all of the exposed people since each had to be contacted individually and appointments made for testing. Contacting the visitors was challenging because of the various geographic locations they came from. Finally, 11 visitors and neonates were lost to follow-up.

Because the results of our study and of earlier studies suggest that few, if any, new infections arise after exposure to an index case of active TB in NICUs, recommendations for appropriate management of $\mathrm{TB}$ exposure in this setting may need to be revised. It is possible that in the modern NICU setting, with incubators, mechanical ventilators and aggressive precautions against exposure to airborne pathogens, neonates may have increased protection against infection. Future recommendations regarding isoniazid therapy in this setting will likely be dictated on the basis of the level of exposure and the severity of disease in the index case.

In summary, we did not document any transmission from the index case to exposed neonates, health care workers or visitors to the NICU. Infants who were at high risk of infection were given isoniazid therapy without adverse sequelae. Although there are published guidelines for TB exposure in pediatrics, there are currently no recommendations for the management of neonates exposed to an active case of TB. From our experience and the few reported cases in the literature, we suggest that neonates with significant exposure to an index case with a high likelihood of transmitting infection (i.e., cavitary TB) be given isoniazid therapy if there are no contraindications. In addition, surveillance for TB infection in specific health care environments should be implemented on a regular basis to prevent active disease. ${ }^{8}$

This article has been peer reviewed.

From the Divisions of Respirology (Sen, Lewis) and Infectious Diseases (Gregson) and the Program in Critical Care Medicine (Sen), University of Western Ontario, London Health Sciences Centre and St. Joseph's Health Centre, London, Ont.

Competing interests: None declared.

Contributors: Mithu Sen conceived the study, was involved in its design, was responsible for data acquisition, analysis and interpretation, and drafted and critically revised the article. James Lewis was responsible for data acquisition, analysis and interpretation, and, with Daniel Gregson, was involved in the design and critical revision of the article. All of the authors gave final approval of the article submitted for publication.

Acknowledgements: We wish to thank the members of the Middlesex-London Health Unit for their assistance with the surveillance and testing of contacts. Parts of this article were presented at the American Thoracic Society's 95th International Conference, held in San Diego in May 2000. 


\section{References}

1. Tuberculosis. Geneva: World Health Organization. Available: www.who.int /gtb/publications/healthcare/introduction.html (accessed 2005 Apr 21).

2. Burk JR, Bahar D, Wolf FS, Green, J, Bailey WC. Nursery exposure of 528 newborns to a nurse with pulmonary tuberculosis. South Med f 1978;71(1):7-10.

3. American Thoracic Society. Targeted tuberculin testing and treatment of latent tuberculosis infection. Am 7 Respir Crit Care Med 2000;161;S221-47.

4. American Academy of Pediatrics, Peter G, ed. Tuberculosis. In: Red book: Report on the Committee on Infectious Disease. Elk Grove Village, IL: American Academy of Pediatrics; 1997.

5. American Thoracic Society. Treatment of tuberculosis and tuberculosis infection in adults and children. Am 7 Respir Crit Care Med 1994;149:1359-74.

6. Maloney SA, Person ML, Gordon MT, Del Castillo R, Boyle JF, Jarvis WR. Efficacy of control measures in preventing nosocomial transmission of multidrug-resistant tuberculosis to patients and health care workers. Ann Intern Med 1995;122:90-5.

7. Bate TWP, Sinclair RE, Robinson MJ. Neonatal tuberculosis. Arch Dis Child
$1986 ; 61: 512-4$

8. Light IJ, Saidleman M, Sutherland JM. Management of newborns after nursery exposure to tuberculosis. Am Rev Respir Dis 1974;109(4):415-9.

9. Steiner P, Rao M, Victoria MS, Rudolph N, Buynoski G. Miliary tuberculosis in two infants after nursery exposure: epidemiologic, clinical, and laboratory findings. Am Rev Respir Dis 1976;113:267-71.

10. Lee LH, LeVea CM, Graman PS. Congenital tuberculosis in a neonatal intensive care unit: case report, epidemiological investigation, management of exposures. Clin Infect Dis 1998;27:474-7.

11. Lewis DB, Wilson CB. Developmental immunology and role of host defenses in neonatal susceptibility to infection. In: Remington JS, Klein JO, eds. Infectious diseases of the fetus and newborn infant. 4th ed. Philadelphia: W.B. Saunders; 1995. p. 20-98.

Correspondence to: Dr. Mithu Sen, London Health Sciences Centre, South Street Site, Rm. N480, 375 South St., London ON

N6A 4G5; fax 519 667-6698; mithu.sen@Ihsc.on.ca

\section{Writing for CMAJ}

CMAJ publishes papers that advance the understanding of medicine and health care, stimulate debate, educate and entertain. Most submissions arrive unsolicited, and we welcome them. Contributors should consult the instructions for authors on eCMAJ (www.cmaj.ca/misc/ifora.shtml).

Research articles report original findings of interest to a general medical audience. Articles should be no more than 2500 words long. Novel case reports will also be considered for the Research section. Research letters are short (750 words) reports that do not require extensive elaboration with respect to methods and results.

Although most Commentaries are written at our invitation, we welcome unsolicited submissions and suggestions for topics and contributors. Commentaries require a succinct and confident style, a clear point of view and a degree of balance.

The Review section contains comprehensive, peerreviewed summaries of recent advances in basic science or clinical practice (systematic and narrative reviews). Most submissions should be no longer than 2000 words.

Analysis provides authoritative articles (1000 words) that explain and contextualize health-related topics. Practice contains pragmatic articles that present or review information for physicians in practice. Prospective authors should contact the editors to discuss ideas and projects.
The Left Atrium gives readers room for reflection. Book and film reviews are solicited by the editor. We welcome unsolicited poetry, fiction and creative nonfiction. The writing should be candid, but patient confidentiality must be respected. If you would like to be added to our list of book reviewers or to discuss ideas for contributions, please contact the senior deputy editor, Anne Marie Todkill.

Letters to the Editor commenting on an article should be entered on our Web site as eLetters at www.cmaj.ca within 8 weeks of the article's appearance; all eLetters are considered for print publication. Submissions should be no more than 250 words long and appropriately referenced. The most effective letters usually raise a single, tersely argued point. Authors whose work is discussed are given an opportunity to respond.

For the unabridged version of Writing for CMAJ, see our Web site at www.cmaj.ca

\section{Editor, CMAJ}

John Hoey, MD

$800663-7336 \times 2118$

john.hoey@cma.ca

To contact other CMAJ staff members, please consult the masthead at the front of the journal or send an email message to pubs@cma.ca 